\title{
Campus numérique et innovation pédagogique : l'hypothèse de la territorialisation
}

\author{
Didier Paquelin* - Jacques Audran** - Hugues Choplin*** \\ Svitlana Hryshchuk ${ }^{* * * *}$ — Stéphane Simonian****** \\ *Université Michel de Montaigne, Bordeaux 3 - IMAGINES (EA2959) \\ Domaine Universitaire-F-33607 Pessac cedex \\ paquelin@u-bordeaux3.fr \\ **Université de Haute Alsace, Mulhouse, CUFEF/SERFA \\ 17, rue de Alfred Werner - 68093 Mulhouse cedex \\ jacques.audran@uha.fr \\ ***Université de Technologie de Compiègne \\ COSTECH - Formation continue \\ BP 20529 - 60205 Compiègne cedex \\ choplin@utc.fr \\ ****Université Paris 8, GRAME \\ Bd de la Liberté - 93210 Saint Denis \\ svitlana.hryshchuk@wanadoo.fr \\ *****Université de Provence, laboratoire ADEF \\ 1, avenue de Verdun - 13140 Lambesc \\ s.simonian@educaix.com
}

\begin{abstract}
RÉSUMÉ. La question de la relation entre l'innovation pédagogique et le développement des campus numériques est au cour d'un projet de recherche labellisé "ERTe ». Une première analyse des pratiques ne semble pas confirmer la relation entre l'introduction d'objets techniques et la transformation des pratiques sociales au sein de l'enseignement supérieur universitaire. Pour comprendre le processus d'innovation nous cherchons à identifier en quoi et comment les espaces-temps, les collectifs et les objectifs participent ou non à ces transformations. Les premiers travaux empiriques conduits ne semblent pas invalider ces hypothèses mais donnent à penser que les processus d'innovation pédagogique et l'impact de ces trois dimensions sont subordonnés à la rencontre de deux "mondes": à la constitution de ce que nous proposons d'appeler un "inter-monde ». Éloignée d'une approche déterministe, l'équipe de recherche proposel'hypothèse selon laquellece processus s'inscrirait dans une dynamique de territorialisation, qui par ses dimensions symboliques et contenantes, organiserait des reconfigurations des pratiques situées de formation.
\end{abstract}

D\&S - 4/2006. Campus numériques 2, pages 365 à 395 
ABSTRACT. The question of a link between the pedagogical innovation and the development of virtual campuses is in the focus of research project developed by an ERTe. The primary study of existing practices doesn't seem to confirm the direct link between the introduction of technological devices and transformation of existing educational approach inside the university higher education system. To understand the innovation process in the universities, we try to identify in what and how such concepts as spaces-times, collectives and technological devices are involved (or not) in these transformations. The first empirical results collected doesn't seem to revoke this approach but provide the prosperous field of reflection. The processes of a pedagogical innovation and the impact of these three concepts could be subordinated to the phenomenon of the two world meeting up during the construction of what can be called "the inter-world". Far from the determinism approach the research team propose hypothesis according to which this process enter into dynamics of territorialization. This dynamics by its symbolical dimensions and cotenants, would organize reconfigurations of the education practice.

MOTS-CLÉS : campus numérique, innovation pédagogique, inter-monde, territorialisation. KEYWORDS : virtual campuses, pedagogical innovation, “inter-world”, territorialization.

\section{Les terrains}

\begin{tabular}{|c|c|}
\hline \multicolumn{2}{|r|}{$\begin{array}{l}\text { ENFI } \\
\text { Environnement Numérique de la Formation Initiale }\end{array}$} \\
\hline Finalité(s) & $\begin{array}{l}\text { Favoriser l'intégration des TICE dans les pratiques des enseignants de } \\
\text { Telecom Paris (ENST). }\end{array}$ \\
\hline Équipe-Projet & $\begin{array}{l}\text { Département Innovation Pédagogique, Service Informatique et } \\
\text { AudioVisuel, Service Études et Programmes, les départements } \\
\text { d'enseignement de l'école. }\end{array}$ \\
\hline $\begin{array}{l}\text { Public(s) cibles } \\
\text { enseignants }\end{array}$ & $\begin{array}{l}\text { Les enseignants chercheurs des départements d'enseignement de } \\
\text { l'école (120). }\end{array}$ \\
\hline $\begin{array}{l}\text { Public(s) cibles } \\
\text { apprenants }\end{array}$ & Les élèves ingénieurs de l'ENST (1000). \\
\hline $\begin{array}{l}\text { Origine du } \\
\text { projet }\end{array}$ & $\begin{array}{l}\text { 1/ Une stratégie de l'école vers l'usage des TICE et l'innovation } \\
\text { pédagogique (2001). } \\
\text { 2/ Un débat interne à l'école sur le thème « Mise en ligne des } \\
\text { ressources pédagogiques de l'ENST, avantages/inconvénients », } \\
\text { impliquant l'ensemble des acteurs (élèves, enseignants, personnels } \\
\text { administratifs et techniques et direction) (2001-2002). }\end{array}$ \\
\hline
\end{tabular}




\begin{tabular}{|c|c|}
\hline & $\begin{array}{l}\text { 3/ L'environnement Numérique de la Formation Initiale (sous- } \\
\text { ensemble de l'Environnement Numérique de Travail de Télécoms } \\
\text { Paris) est assemblé, construit, à partir des besoins et de l'évolution } \\
\text { des pratiques des enseignants et des élèves. L'agrégation des projets } \\
\text { «portail de la formation initiale » et « système d'information » sous } \\
\text { le nom de ENFI est donc le résultat d'une volonté institutionnelle } \\
\text { (étape } n^{\circ} 1 \text { ) associé à l'articulation d'outils construits sur les besoins } \\
\text { des enseignants. }\end{array}$ \\
\hline $\begin{array}{l}\text { Stratégie } \\
\text { d'action }\end{array}$ & $\begin{array}{l}\text { 1/ Passage d'une stratégie d'actions visant les enseignants } \\
\text { «pionniers » à une stratégie d'accompagnement de l'ensemble des } \\
\text { enseignants de l'ENST. } \\
\text { 2/ Déploiement d'« environnements » de travail articulés et cohérents } \\
\text { concernant les aspects administratif (admission, scolarité) et } \\
\text { pédagogique (capitalisation et diffusion des ressources pédagogiques, } \\
\text { mise en place de services pédagogiques innovants, articulation aux } \\
\text { pratiques pédagogiques existantes). } \\
\text { 3/ Mise en place d'un accompagnement par étape, respectant le } \\
\text { rythme de l'enseignant et ses demandes. Accompagnement d'équipes } \\
\text { pédagogiques (et non d'enseignants). } \\
\text { 4/ Valorisation des travaux pédagogiques de l'enseignant et de l'élève. }\end{array}$ \\
\hline $\begin{array}{l}\text { Ingénierie } \\
\text { financière du } \\
\text { projet }\end{array}$ & $\begin{array}{l}\text { Ressources humaines : Département Innovation Pédagogique, } \\
\text { Service Informatique et Audio Visuel. } \\
\text { Budgets spécifiques aux projets Portail FI, SIFI et ENFI (financement } \\
\text { de personnes en CDD). }\end{array}$ \\
\hline Innovation & $\begin{array}{l}\text { Appréhender l'innovation comme un processus progressif et } \\
\text { accompagné par lequel il y a passage des expérimentations des TICE } \\
\text { aux usages et à l'évolution des pratiques formatrices. } \\
\text { L'Environnement Numérique de la Formation INitiale doit permettre } \\
\text { d'induire un changement dans les pratiques pédagogiques des } \\
\text { enseignants en pérennisant l'utilisation des TIC. }\end{array}$ \\
\hline Contenu & L'ensemble des domaines scientifiques et techniques de l'école. \\
\hline $\begin{array}{l}\text { Niveau de } \\
\text { formation }\end{array}$ & Première, deuxième et troisième année d'ingénieur. \\
\hline $\begin{array}{l}\text { Durée de la } \\
\text { formation }\end{array}$ & Tout au long des trois années de formation. \\
\hline $\begin{array}{l}\text { Modèles } \\
\text { pédagogiques }\end{array}$ & $\begin{array}{l}\text { En fonction des spécificités de l'Unité d'Enseignement et des } \\
\text { positionnements de l'enseignant et de son souhait d'expérimentation } \\
\text { (souple et évolutif). }\end{array}$ \\
\hline Plate-forme & $\begin{array}{l}\text { Extranet institutionnel de l'école (EOLE) et sites Internet } \\
\text { institutionnels (ENST, ParisTech, GET-Groupe des Écoles des } \\
\text { Telécommunications). }\end{array}$ \\
\hline Acteurs & $\begin{array}{l}\text { Tous les acteurs (enseignants, élèves et personnels administratifs et } \\
\text { techniques) de l'ENST. }\end{array}$ \\
\hline
\end{tabular}




\begin{tabular}{|c|c|}
\hline \multicolumn{2}{|c|}{$\begin{array}{c}\text { PEGASUS } \\
\begin{array}{c}\text { (Portail de reprise d'Études Graduelles et d'Accès par un Soutien personnalisé } \\
\text { à l'UniverSité) }\end{array}\end{array}$} \\
\hline Finalité(s) & $\begin{array}{l}\text { Développement de l'accès du public à la formation préparatoire du } \\
\text { Diplôme d'Accès aux Études Universitaires (DAEU). }\end{array}$ \\
\hline Équipe-Projet & $\begin{array}{l}\text { Consortium de six universités : Université Nice-Sophia-Antipolis } \\
\text { - chef de projet -, Universités Toulon-Var, Le Havre, Paris 13, } \\
\text { Antilles-Guyane et Haute Alsace Mulhouse Colmar. }\end{array}$ \\
\hline $\begin{array}{l}\text { Public(s) cibles } \\
\text { apprenants }\end{array}$ & $\begin{array}{l}\text { Adultes désirant obtenir l'équivalent du baccalauréat pour reprendre } \\
\text { des études et/ou passer des concours nécessitant l'obtention d'un } \\
\text { niveau IV. Public désirant une remise à niveau dans une matière } \\
\text { particulière (possibilité d'inscription à } 1 \text { seul module). }\end{array}$ \\
\hline $\begin{array}{l}\text { Origine du } \\
\text { projet }\end{array}$ & $\begin{array}{l}\text { Projet retenu dans le cadre de l'appel à projet « Campus numériques », } \\
\text { campagne 2001-2002 (www.campus.pegasus.org). }\end{array}$ \\
\hline $\begin{array}{l}\text { Stratégie } \\
\text { d'action }\end{array}$ & $\begin{array}{l}\text { Conception d'un système original de cours tutorés associés à des } \\
\text { outils de communication simples. }\end{array}$ \\
\hline $\begin{array}{l}\text { Ingénierie } \\
\text { financière du } \\
\text { projet }\end{array}$ & $\begin{array}{l}\text { Groupe de pilotage ( } 2 \text { membres pour chacune des } 6 \text { universités). } \\
\text { Financements Régions-FSE, Conseil Généraux et Universités. }\end{array}$ \\
\hline $\begin{array}{l}\text { État } \\
\text { d'avancement } \\
\text { du projet }\end{array}$ & $\begin{array}{l}\text { Ouverture de l'option Littéraire, dite DAEU A en janvier } 2003 . \\
\text { Ouverture de l'option Scientifique, dite DAEU B en septembre } 2004 .\end{array}$ \\
\hline Innovation & $\begin{array}{l}\text { Concerne plus spécifiquement l'ingénierie de conception du } \\
\text { dispositif. Démarche, qui pourrait par anticipation, apporter une } \\
\text { certaine souplesse permettant la « retouche » (ou l'adaptation) et } \\
\text { favoriser l'activité d'accompagnement. Il s'agit de prévoir dès la } \\
\text { conception la possibilité de régulation de la mise en œuvre. }\end{array}$ \\
\hline Contenu & $\begin{array}{l}\text { Ensemble de modules : français, langues, mathématiques, histoire, } \\
\text { géographie, philosophie, physique, chimie, SVT, méthodologie de } \\
\text { travail en ligne. } \\
\mathbf{2 8} \text { modules de formation sont proposés. } \\
\text { Chaque module ( } 20 \text { séquences d'environ } 2 \text { heures de cours) regroupe } \\
\text { les cours d'une même matière. Les matières principales comprennent } \\
2 \text { modules (soit } 40 \text { séquences) comme français et langues en A } \\
\text { ou maths et physique en B. Le module de méthodologie de travail } \\
\text { comprend } 14 \text { séquences. }\end{array}$ \\
\hline $\begin{array}{l}\text { Niveau de } \\
\text { formation }\end{array}$ & Niveau IV \\
\hline $\begin{array}{l}\text { Durée de la } \\
\text { formation }\end{array}$ & $\begin{array}{l}\text { Définie par décret minimalement à } 225 \text { heures (l'arrêté du } 3 \text { août } 1994 \\
\text { fixant le nombre d'heures d'enseignement minimum ; article 6) }\end{array}$ \\
\hline
\end{tabular}




\begin{tabular}{|l|l|}
\hline $\begin{array}{l}\text { Modèles } \\
\text { pédagogiques }\end{array}$ & $\begin{array}{l}\text { Cours multimédias assistés par tutorat et accompagnement. } \\
\text { Proposer autre chose que des polycopiés en ligne : enrichissement via } \\
\text { du multimédia interactif. } \\
\text { Des « devoirs » (3 par module), sont à rendre aux tuteurs tout au long } \\
\text { du cursus. Des regroupements (3 par an) sont organisés. }\end{array}$ \\
\hline Plate-forme & $\begin{array}{l}\text { Initialement plate forme WebCT hébergée par le Centre National } \\
\text { d'Enseignement à Distance (partenariat éditorial). }\end{array}$ \\
\hline Acteurs & $\begin{array}{l}\text { Universités partenaires + CNED + Ateliers de Pédagogie } \\
\text { Personnalisée + Point d'Accès à la Téléformation (P@T). } \\
\text { Août 2004, évolution du consortium initial qui s'est notamment } \\
\text { concrétisée par le départ du CNED. }\end{array}$ \\
\hline
\end{tabular}

\begin{tabular}{|c|c|}
\hline \multicolumn{2}{|c|}{$\begin{array}{l}\text { SCENARI sup (Système de Conception des Enseignements Numériques Adaptables, } \\
\text { Réutilisables et Interactifs pour l'enseignement supérieur public) }\end{array}$} \\
\hline Finalité(s) & $\begin{array}{l}\text { Développer un outil de gestion de production de contenu avec des } \\
\text { outils issus de l'ingénierie documentaire pour développer des usages } \\
\text { selon une « approche organisationnelle » incrémentale. }\end{array}$ \\
\hline Équipe-Projet & $\begin{array}{l}\text { Équipe Formation Continue de l'Université Technologie de } \\
\text { Compiègne : unité d'innovation des contenus et des services qui } \\
\text { assure une fonction de transfert. } \\
\text { Démarche mise en œuvre avec des partenaires industriels : AXA } \\
\text { (Assurances), PSA (Peugeot Citroën), SNCF (transport ferroviaire). }\end{array}$ \\
\hline $\begin{array}{l}\text { Public(s) cibles } \\
\text { enseignants }\end{array}$ & Enseignants pour leur expertise pédagogique et de contenu. \\
\hline $\begin{array}{l}\text { Public(s) cibles } \\
\text { apprenants }\end{array}$ & Pas directement concerné dans la chaîne de production des contenus. \\
\hline $\begin{array}{l}\text { Origine du } \\
\text { projet }\end{array}$ & $\begin{array}{l}\text { Depuis 1999, développement d'un outil de numérisation de documents } \\
\text { pédagogiques et d'une chaîne de production associée, reposant sur } \\
\text { une ingénierie pédagogique et organisationnelle spécifique visant à } \\
\text { faire accepter l'outil par les différents acteurs. }\end{array}$ \\
\hline $\begin{array}{l}\text { Stratégie } \\
\text { d'action }\end{array}$ & $\begin{array}{l}\text { L'un des axes stratégiques d'action est fondé sur une démarche } \\
\text { incrémentale structurante de l'ingénierie pédagogique et } \\
\text { organisationnelle. }\end{array}$ \\
\hline $\begin{array}{l}\text { Ingénierie } \\
\text { financière du } \\
\text { projet }\end{array}$ & Ministère délégué à la Recherche + Établissements. \\
\hline
\end{tabular}




\begin{tabular}{|c|c|}
\hline $\begin{array}{l}\text { État } \\
\text { d'avancement } \\
\text { du projet }\end{array}$ & $\begin{array}{l}\text { Les différents partenaires ont été formés à la méthodologie à l'outil } \\
\text { SCENARIchain v1.0 (mars 2004). La chaîne éditoriale a ainsi pu être } \\
\text { déployée dans les différents établissements. } \\
\text { Par exemple, l'Université de Rennes } 1 \text { a produit } 3 \text { modules } \\
\text { (estimation : } 30 \text { à } 40 \text { h travail apprenant par module) pour le } \\
\text { lancement d'un DUT-GEA (Gestion des Entreprises et des } \\
\text { Administrations) à distance. } \\
\text { Autre exemple, l'Université d'Aix Marseille } 2 \text { a, quant à elle, effectué } \\
\text { la production de } 8 \text { modules correspondant à } 120 \text { h d'enseignement } \\
\text { dans le cadre du projet euroméditerranéen INTERREG IIIB et d'un } \\
\text { projet interne de mise à distance sur des contenus de type sciences } \\
\text { économiques. La Cellule Innovation Pédagogique de l'université } \\
\text { prévoit la finalisation d'une vingtaine de modules. } \\
\text { Dernier exemple, l'Université de Corte a produit } 9 \text { modules } \\
\text { correspondant à environ } 120 \text { h d'enseignement. } 4 \text { de ces modules } \\
\text { concernent le Certificat Informatique Internet (C2I) et les } 5 \\
\text { autres couvrent des champs disciplinaires variés : comptabilité, } \\
\text { informatique, escalade... }\end{array}$ \\
\hline Innovation & $\begin{array}{l}\text { L'innovation concerne plus spécifiquement l'approche de la } \\
\text { structuration des documents numériques. }\end{array}$ \\
\hline $\begin{array}{l}\text { Modèles } \\
\text { pédagogiques }\end{array}$ & $\begin{array}{l}\text { Si les concepteurs de l'outil Scenari semblent estimer que l'outil } \\
\text { peut être utilisé dans diverses perspectives pédagogiques, les usages } \\
\text { actuels paraissent largement privilégier une pédagogie centrée sur le } \\
\text { document. }\end{array}$ \\
\hline Plate-forme & Développement d'outils de spécification de contenus numériques. \\
\hline Acteurs & $\begin{array}{l}\text { Cet environnement sera constitué d'un système informatique à } \\
\text { déployer en milieu universitaire au niveau de la formation initiale } \\
\text { accompagné d'une méthodologie de production de contenus } \\
\text { et de recommandations organisationnelles fondées sur les } \\
7 \text { expérimentations menées au sein des universités de Compiègne } \\
\text { (UTC), de Corte, d'Aix-Marseille 2, de Nancy } 2 \text { (Videoscop), de } \\
\text { Rennes } 1 \text { et de Bordeaux } 1 \text { et de l'École Nationale Supérieure des } \\
\text { Télécoms (ENST). }\end{array}$ \\
\hline
\end{tabular}

\begin{tabular}{|l|l|}
\hline \multicolumn{2}{|c|}{ (Technologies Éducatives pour le Management de l'Information et des Savoirs) } \\
\hline Finalité(s) & $\begin{array}{l}\text { Développement d'une stratégie d'innovation pédagogique par les } \\
\text { TICE. }\end{array}$ \\
\hline Équipe-Projet & $\begin{array}{l}\text { Université de Technologie de Troyes (UTT)/ Centre d'Innovation } \\
\text { Pédagogique (CIP) }\end{array}$ \\
\hline $\begin{array}{l}\text { Public(s) cibles } \\
\text { enseignants }\end{array}$ & $\begin{array}{l}\text { Enseignants tronc commun, enseignements en branches ingénieurs et } \\
\text { enseignants en master pro et recherche. }\end{array}$ \\
\hline $\begin{array}{l}\text { Public(s) cibles } \\
\text { apprenants }\end{array}$ & idem \\
\hline
\end{tabular}




\begin{tabular}{|c|c|}
\hline $\begin{array}{l}\text { Origine du } \\
\text { projet }\end{array}$ & $\begin{array}{l}\text { Depuis 2001, l'UTT s'est engagée dans une stratégie d'innovation par } \\
\text { les TICE. } \\
\text { Depuis 2002, l'UTT, via son CIP, a mis en place TEMIS dont l'un des } \\
\text { objectifs est d'offrir des informations, des ressources, des services, } \\
\text { des outils et des applications en ligne pour l'apprentissage. }\end{array}$ \\
\hline $\begin{array}{l}\text { Stratégie } \\
\text { d'action }\end{array}$ & $\begin{array}{l}\text { Le développement du portail est inscrit dans une stratégie } \\
\text { d'innovation par le changement culturel touchant l'UTT dans } \\
\text { son ensemble. Le choix est porté sur les activités d'apprentissage } \\
\text { (notamment collaboratives) plutôt que sur la scénarisation de } \\
\text { contenus. }\end{array}$ \\
\hline $\begin{array}{l}\text { Ingénierie } \\
\text { financière du } \\
\text { projet }\end{array}$ & $\begin{array}{l}\text { Financement interne et financement régional. } \\
\text { Depuis 2005, cette action est un programme inscrit dans la } \\
\text { dynamique de la LOLF, au même titre que l'enseignement et la } \\
\text { recherche. }\end{array}$ \\
\hline $\begin{array}{l}\text { État } \\
\text { d'avancement } \\
\text { du projet }\end{array}$ & $\begin{array}{l}\text { L'ensemble des plateformes et des outils prévu est opérationnel et } \\
\text { touche } 100 \% \text { des étudiants et } 50 \% \text { des enseignants (qui déposent ou } \\
\text { accèdent à des ressources pédagogiques en ligne). } \\
\text { Sur la seule plateforme Webct, plus de } 60 \text { modules ont été développés } \\
\text { en } 3 \text { ans, soit } 37 \% \text { des enseignants de l'UTT et } 85 \% \text { des étudiants } \\
\text { sont concernés. }\end{array}$ \\
\hline Innovation & $\begin{array}{l}\text { L'UTT associe à l'ingénierie traditionnelle de la FOAD, une réflexion } \\
\text { sur les dimensions humaines, gestionnaires et organisationnelles du } \\
\text { changement lié à la mise en œuvre d'un dispositif innovant. }\end{array}$ \\
\hline Contenu & Lié aux enseignements de l'UTT \\
\hline $\begin{array}{l}\text { Niveau de } \\
\text { formation }\end{array}$ & $\begin{array}{l}\text { Près de } 100 \% \text { du marché interne du CIP est la formation initiale. } \\
\text { L'évolution en cours concerne l'émergence d'une activité de transfert } \\
\text { auprès des industriels et plus largement d'entreprises utilisatrices de } \\
\text { e-learning. }\end{array}$ \\
\hline \multicolumn{2}{|l|}{$\begin{array}{l}\text { Durée de la } \\
\text { formation }\end{array}$} \\
\hline $\begin{array}{l}\text { Modèle } \\
\text { pédagogique }\end{array}$ & $\begin{array}{l}\text { Centré sur les activités d'apprentissage et plus spécifiquement sur la } \\
\text { dimension collaborative de celles-ci. } \\
\text { Initialement au format papier, ces ressources sont numérisées et } \\
\text { progressivement enrichies via des animations, des exercices pour les } \\
\text { rendre interactives. Les ressources peuvent ensuite évoluer pour être } \\
\text { utilisée à distance en remplacement de cours présentiels. }\end{array}$ \\
\hline Plate-forme & $\begin{array}{l}\text { TEMIS est un portail « métier » d'accès centralisé qui offre un } \\
\text { ensemble d'outils : des plates-formes techniques, des outils auteurs, } \\
\text { des outils techniques }\end{array}$ \\
\hline
\end{tabular}




\begin{tabular}{|l|l|}
\hline Acteurs & $\begin{array}{l}\text { Acteurs internes : la direction, les personnels administratifs et } \\
\text { techniques, les étudiants, les enseignants, les chefs de service } \\
\text { (bibliothèque, recherche, CRI, enseignement, etc.), les responsables } \\
\text { de masters. } \\
\text { Acteurs externes : la Région, le ministère, les entreprises, les campus } \\
\text { numériques, les laboratoires de recherche, les prestataires d'outils, } \\
\text { l'Europe. }\end{array}$ \\
\hline
\end{tabular}

\section{Introduction}

L'alliance de l'ingénierie de formation et de l'informatique donne naissance à des projets et des artefacts étranges auxquels il semble difficile d'attribuer un nom. Que ces entités soient baptisées campus numériques, instruments numériques de gestion des connaissances, plates-formes ou espaces collaboratifs de formation (au point qu'il semble illusoire de tenter ici une énumération), elles ont en commun de se présenter comme des systèmes reposant sur des technologies numériques au service de la formation. Au-delà de cette base étroite, ces systèmes forment une constellation à la cartographie incertaine, tant ils semblent expérimentaux.

Nos difficultés de repérage proviennent sans doute du fait que le fonctionnement des systèmes informatiques, qui n'a cessé de progresser en matière de performances et de fiabilité au fil des années, a nourri tous les espoirs et ouvre actuellement de nombreuses possibilités d'exploration. De manière conjuguée, chacune des quatre dernières décennies a produit son lot de déceptions pour ce qui est de l'application de l'informatique à l'éducation et à la formation. Qu'il s'agisse de l'impasse behavioriste des années 1965-1975 abandonnant le projet d'un enseignement totalement programmé, ou des difficultés à s'imposer rencontrées par le constructivisme piagétien des années Logo (1975-1985), ou encore des succès limités du multimédia éducatif dépassant rarement l'usage individuel ou familial dans les années 1985-1995, et plus récemment des réserves sur l'emploi d'Internet en situation d'enseignement depuis les années 1995, les pratiques éducatives ont toujours semblé en décalage par rapport aux progrès technologiques, et de façon plus alarmante avec les usages professionnels de l'informatique dans la société civile.

Nos nouveaux objets numériques dédiés à l'éducation et à la formation, échapperont-ils à cette fatalité ? On peut légitimement se demander si 2005-2015 ne sera pas la décennie des campus numériques oubliés, des plates-formes de formation surnuméraires et des espaces collaboratifs improductifs. Les quatre décennies précédentes nous ont appris à quel point il fallait accorder de l'importance à la synchronisation du développement des artefacts informatiques et de l'évolution des pratiques sociales des acteurs de la sphère éducative. En un mot, comme pour tout objet qui vise l'adoption sociale par les usages, les potentialités de ces objets numériques sont corrélées aux évolutions des pratiques des usagers. 
Dans cet article, en nous appuyant sur une première analyse empirique de cinq de ces objets étranges au sein de l'enseignement supérieur, nous tenterons de voir si considérer ces entités comme des objets intermédiaires, acteurs d'un système qui dépasse largement l'outil informatique, ne serait pas un moyen de penser autrement ces entités numériques. Nous faisons l'hypothèse que plus que des objets définis par leurs fonctionnalités, ces projets d'ingénierie numérique de formation créent une situation problématique originale au sens où ils sont à la fois les instruments et les objets d'enjeux qui concernent l'organisation des pratiques d'enseignement et n'ont souvent pas grand-chose à voir avec l'informatique.

La question directrice de notre recherche, conduite au sein d'une Équipe de Recherche Technologique en Éducation (ERTe) ${ }^{1}$, est la suivante : à quelles conditions les campus numériques peuvent-ils ou non susciter les processus d'innovation pédagogique au sein d'une institution éducative de l'enseignement supérieur? Questionnés par ce problème, nous parcourrons dans cet article trois temps.

a) Tout d'abord, nous formulerons trois hypothèses touchant le déploiement de ces processus dans les campus numériques. Ces hypothèses supposent que ce déploiement est affecté par trois dimensions : les espaces-temps qu'engagent les campus numériques; les collectifs qui réunissent les apprenants, les enseignants et les acteurs techniques et administratifs de ces campus; les objets (technologiques ou non) qu'ils mobilisent dans leur conception ou leur mise en œuvre ;

b) Nous procéderons ensuite à une $1^{\text {re }}$ analyse et comparaison des 5 terrains $\mathrm{du}$ projet. Les premiers travaux empiriques conduits ne semblent pas invalider ces hypothèses mais donnent à penser que les processus d'innovation pédagogique et l'impact de ces trois dimensions sont subordonnés à la rencontre de deux « mondes »: à la constitution de ce que nous proposons d'appeler un «inter-monde ». C'est cette constitution qui semble aujourd'hui difficile à repérer sur nos différents campus numériques ;

c) Finalement, notre hypothèse sera que la constitution de cet inter-monde procède d'une territorialisation, concept qui constitue l'enjeu théorique de nos travaux à venir. Dans le cadre de cet article, nous proposons ici de la caractériser en l'associant en particulier à l'idée de situation.

\section{Ce que nous cherchons à comprendre}

L'innovation pédagogique est définie, dans ce contexte comme une transformation des pratiques sociales de la formation. À ce titre, l'innovation pédagogique est

1. Équipe labellisée par le Ministère de l'Enseignement Supérieur et de la Recherche sous le numéro ERTe 59 et intitulée "Campus Numérique et Innovation Pédagogique ». Ce projet est porté initialement par l'Université de Provence, l'Université Michel de Montaigne - Bordeaux 3 et l'École Nationale des Telecom - Paris. Une équipe d'une vingtaine de chercheurs francophones a été constituée. 
repérable par des indices de mises en mouvement du système initial. Notre recherche repose sur le postulat de l'existence d'un processus spécifique d'appropriation, deuxième temps de l'innovation selon Alter $(2000)^{2}$ après l'invention et avant l'institutionnalisation, par lequel les acteurs donnent sens au dispositif lors d'une dynamique singulière qui à la fois s'inscrit dans des pratiques sociales existantes et crée de nouvelles pratiques. Nous nous référons explicitement à la notion d'appropriation proposée par Honoré (2001, p. 245)3 , pour qui « s'approprier signifie intégrer quelque chose dans son expérience (un fait, un événement, une situation, une connaissance, une technique...) par sa compréhension, donc par le sens qui lui est donné, en le rapportant à ce qui nous concerne, à ce qui nous soucie. L'appropriation de ce que nous comprenons est rendue possible par son explication qui la rapporte à des significations préalablement acquises en les confirmant, en les transformant ou en les complétant par de nouvelles significations ». Notre propos n'est pas de nous intéresser à l'appropriation d'un objet technique par un individu, mais bien de comprendre comment trois entités, les espaces-temps, les collectifs et les objets, participent de ce processus expérientiel de transformation des pratiques sociales.

L'innovation, comme traduction de l'appropriation, supposerait des transformations des pratiques sociales « consécutives » à une modification structurelle, c'est-àdire une modification des règles et des ressources qui organisent habituellement l'action. Pour analyser et comprendre ces modifications nous étudierons les recombinaisons des composantes spatio-temporelles et sociales des pratiques de l'acte de formation et la fonction des objets techniques dans leur triple dimension macro (l'institution), méso (l'équipe) et micro (l'apprentissage), cela tant au niveau de la conception que de la mise en œuvre des dites pratiques. L'analyse de ce mouvement, éclairera la trajectoire4 des transformations par lesquelles se construisent les usages.

Nous faisons l'hypothèse opérationnelle et générale suivante : la prise en compte ou l'intégration de trois pôles que sont l'organisation, l'enseignant et l'apprenant pose des problèmes déterminants dans la conception et dans la mise en ouvre des campus numériques.

Par « campus numérique » nous désignons une entité visant la formation qui réponde aux trois caractéristiques spécifiques suivantes :

- elle se déploie dans l'enseignement supérieur ;

2. Alter N., L'innovation ordinaire, Paris, PUF, 2000.

3. Honoré B., Soigner, Paris, Seli Arslan, 2001.

4. Pour Serge Proulx (2002), l'expression «trajectoire d'usages» désigne «les parcours singuliers que les individus empruntent à travers la constellation d'objets communicationnels passés, présents ou émergents qui leur sont offerts et qui constituent un environnement informationnel et cognitif privilégié dans l'élaboration de leurs pratiques d'information et de communication ». 
- elle mobilise l'usage des technologies de l'information et de la communication ;

- elle désigne une formation ouverte et à distance en cela qu'elle articule des temps de formation en présence et d'autres à distance.

Ces Campus Numériques ont pour principale mission le développement d'une offre de formation ouverte et à distance (FOAD) qui permette par des méthodes pédagogiques adaptées d'organiser la formation des étudiants de manière asynchrone ou synchrone, sur place ou à distance. Les analyses de Kaye $(1988)^{5}$ et de Glikman (1994) ${ }^{6}$ caractérisent la formation à distance comme un dispositif ayant recours aux médias (presse écrite, radio et télévision) en tant qu'élément majeur de l'apprentissage. La formation à distance permet un affranchissement des contraintes spatiales et temporelles propres aux dispositifs d'apprentissage en présence d'un formateur. Aujourd'hui, deux types de discours justifieraient le recours à la formation à distance. Le premier met l'accent sur « la démocratisation de l'accès à la formation : l'individu, où qu'il soit, quelles que soient sa situation sociale et économique, ses conditions de vie et ses inhibitions, se verra proposé un enseignement spécifique et individualisé. Le second considère que l'enjeu de ce type de dispositif est d'ordre économique : la standardisation de l'offre de formation permet de réduire considérablement les coûts de production ainsi que ceux du suivi des bénéficiaires » (Bissey \& Moreau, 2003, p. 20-21) ${ }^{7}$. Cette dernière considération peut être un des éléments explicatifs de la difficulté d'intégrer les campus numériques au sein des institutions éducatives et formatives.

Nous considérons, dans cette perspective, que le dispositif « campus » désigne à la fois « un lieu social d'interaction et de coopération » ${ }^{8}$ et l'« objet » même de cette interaction, coopération ou encore négociation ${ }^{9}$. En ce sens, un campus numérique constitue un dispositif si, lors de sa conception et/ou de sa mise en œuvre, émerge un «lieu $»^{10}$ favorisant, entre ses acteurs (apprenants compris), les négociations et/ ou les régulations de certaines de ces composantes (telles par exemple que l'ordre ou les objectifs de certaines des situations d'apprentissage proposées). L'hypothèse

5. Kaye A., «L'enseignement à distance : un état de la question ». Perspectives, vol. XVIII, $\mathrm{n}^{\circ} 1$. pp. 41-54, 1988.

6. Glikman, V., « Formation à distance », in Dictionnaire encyclopédique de l'éducation et de la formation, P. Champy \& C. Etévé (dir), Paris, Nathan, pp. 441-446, 1994.

7. Bissey C., Moreau J.-L., TIC et NET, Nouvelles voies pour la formation, PUF, 2003.

8. Peraya D., « Médiation et médiatisation : les campus virtuels. » in Jacquinot-Delaunay G. et Monnoyer L. (dir.) Le dispositif entre usage et concept, CNRS, Hermes 25, pp. 153-167, 1999.

9. Jacquinot-Delaunay G. et Choplin H., «La démarche dispositive aux risques de l'innovation », Éducation permanente, nº 152, pp. 185-198, 2002.

10. Plus exactement: un espace-temps social. Cf. Paquelin D. (dir.). Analyse systémique des usages des technologies de l'information et de la communication pour l'apprentissage, CNCRE, rapport final, 2000. Notre description du dispositif fait ainsi signe vers les trois dimensions que nous privilégions dans le projet pour le problématiser : les espaces-temps, les collectifs et les objets. 
de ces travaux est que l'émergence de ce lieu et de cet objet ${ }^{11}$ est susceptible de cristalliser le processus d'innovation pédagogique.

\section{Description des terrains}

La mise en place des campus numériques participe de cette dynamique qui fait de l'innovation une valeur fondatrice de la modernisation de l'institution universitaire. Les discours politiques expriment explicitement cette volonté : «La Formation Ouverte, doit permettre à l'enseignement supérieur de se rénover et d'asseoir sa présence au niveau international $\rangle^{12}$. Interroger la relation entre leur mise en place et l'innovation pédagogique dans l'enseignement supérieur, conserve à distance toute son actualité. Ces campus expriment certains principes de l'industrialisation ${ }^{13}$ tels que l'idéologisation, la technologisation et la rationalisation. Pour traiter cette question centrale cinq campus numériques spécifiques et complémentaires constituent nos « terrains » ${ }^{14}$ d'analyse ${ }^{15}$ :

- PEGASUS (Portail de reprise d'Études Graduelles et d'Accès par un Soutien personnalisé à l'UniverSité), mis en place par un consortium de six universités ${ }^{16}$ dès 2002, est un dispositif de formation à distance, conçu afin de donner la possibilité d'obtenir un diplôme d'accès aux études universitaires à un public qui n'a pas eu le bac.

11. Cros F. (dir.), Dynamiques du changement en éducation et en formation, Versailles, INRP/ IUFM, 1998.

12. Propos tenu lors du lancement de l'un des appels d'offres «Campus Numérique ». Le Monde daté du samedi 30 septembre 2000.

13. Tels que les a rappelé Moeglin (Entretien avec, «À la recherche de l'industrialisation du tutorat à distance ", in Distances et Savoirs Vol. 2 n 2/2005 p. 252, 2005).

14. La notion de terrain, entité délimitant la portée d'une recherche, est souvent peu explicitée, nous la relions ici à l'idée de territorialité symbolique. Nos terrains constituent donc à la fois une délimitation «naturelle» de nos recherches, mais aussi une façon ethnographique de traduire, via une stratégie textuelle (Geertz, 1996), les particularités, telles que les acteurs les « expriment», de ces entités hybrides (à la fois espaces-temps, collectifs humains et objets) que sont les campus numériques. La pluralité de ces mondes anthropologiques (Affergan, 1997) sera également utile pour rendre compte de cette territorialisation particulière.

Les terrains sont présentés en début d'article.

15. Ces analyses ont débuté sur les différents terrains depuis janvier 2006. La phase d'investigation (étude des documents historiques des projets, entretiens semi-directifs avec différents acteurs engagés dans le projet) se poursuivra jusqu'à juin 2007. Des séminaires réguliers entre les membres de l'équipe ERTe participent à l'élaboration du questionnement et la construction d'outils méthodologiques, situant ce projet dans la dynamique spécifique de recherche-action. La présente contribution a été l'objet de nombreuses discussions et parfois de divergence au sein du groupe de recherche.

16. Université Nice Sophia-Antipolis, Universités Toulon-Var, Le Havre, Paris 13, AntillesGuyane et Haute Alsace Mulhouse Colmar. 
- TEMIS (Technologies Éducatives pour le Management de l'Information et des Savoirs), mis en place par le Centre d'Innovation Pédagogique de l'Université de Technologie de Troyes depuis 2002, est un portail au service des enseignants qui promeut les pratiques de formation en ligne. Il offre des informations, des ressources, des services, des outils et des applications en ligne pour l'apprentissage.

- ENFI (Environnement Numérique de la Formation Initiale), mis en place par le département Innovation Pédagogique de Télécom Paris, est un portail au service des étudiants et des enseignants; il est accessible via l'extranet/intranet de l'école. Initié en 2001, il accompagne les enseignants dans la «conception » et l'usage de sites pédagogiques collectifs.

- SCENARISUP (Système de Conception des Enseignements Numériques Adaptables, Réutilisables et Interactifs pour l'enseignement supérieur public), mis en place dès 1999 par l'équipe Formation Continue de l'Université Technologique de Compiègne en partenariat avec plusieurs autres universités ${ }^{17}$, est un outil de numérisation et de gestion de documents pédagogiques pour l'instauration d'une nouvelle pratique organisationnelle de la production de documents pédagogiques dans l'université (passage d'une organisation informelle à une organisation rationalisée).

Un cinquième terrain, au statut particulier dans notre recherche, complète ce panel : le Campus Virtuel Suisse (CVS) dont la mission est de favoriser le développement de pratiques d'apprentissage et d'enseignement avec les Technologies de l'Information et de la Communication dans l'enseignement supérieur suisse. Ce n'est pas le CVS en tant que tel qui est étudié, mais le travail de soutien auprès de seize projets de mise à distance de cours universitaires, mené par le groupe IntersTICES auquel lui a succédé le réseau GIRAFE en 2004. L'approche de type recherche-action-formation, qui a par ailleurs été analysée par l'équipe GIRAFE et donné lieu à de multiples publications, intéresse directement l'ERTe en cela qu'elle traite, du moins c'est son projet, de la relation entre la recherche et l'action, cherchant tout à la fois à lever des verrous opérationnels et à maintenir une posture distanciée propre à la recherche.

17. Le projet SenariSup a été lancé en novembre 2003 sur un financement du Fonds Recherche et Technologie du ministère de l'Éducation nationale. Suite à la demande du ministère, l'environnement Scenari est adapté aux contraintes des établissements de l'enseignement supérieur. Afin de prendre en compte ces contraintes, la chaîne éditoriale ScenariSup est déployée au sein de 10 établissements, pour la plupart par l'intermédiaire des cellules TICE : Universités de Rennes 1 (2003), Bordeaux (2003), Nancy 2 (2003), Aix-Marseille 2 (2003), UTC (2003), Corte en janvier 2004, Paris 11 et Valenciennes en octobre 2004, Paris 1 et Rouen en septembre 2005. 


\section{Première analyse des terrains}

Nous pouvons aborder l'analyse comparative de ces cinq terrains en distinguant en premier lieu les trois caractéristiques ou les trois niveaux suivants :

- la stratégie de développement (ou stratégie "partenariale ») adoptée (en fonction des objectifs poursuivis et du positionnement du campus numérique relativement à l'organisation ou aux partenaires) (niveau macro) ;

- le choix de l'organisation du travail qui exprime le degré de rationalisation et la production des biens et des services : « $\operatorname{artisanat}^{18} »$ ou tendance à l'industrialisation ? (niveau méso) ;

- le rapport à l'enseignant-chercheur, acteur qui paraît en effet déterminant du point de vue des processus de changement susceptibles de se déployer dans ces bureaucraties professionnelles ${ }^{19}$ que sont nos universités (la stratégie adoptée par les concepteurs se base-t-elle sur son adhésion ou sur sa participation collaborative ?) (niveau micro).

Au niveau macro tout d'abord, le projet PEGASUS a pour objectif de mettre en place un dispositif de formation à distance commun à plusieurs universités avec, à la clé pour les étudiants, l'obtention du Diplôme d'Accès aux Études Universitaires ${ }^{20}$ fourni par l'établissement où a été effectuée l'inscription. La complexité de la gestion administrative partagée entre plusieurs entités appartenant à l'enseignement supérieur, l'utilisation mutualisée du contenu, des services et des moyens de diffusion de la formation à distance, ne permet pas une localisation de ce campus numérique au sein d'un établissement précis. Le choix initial de construction du consortium et la recherche de pratiques communes à plusieurs universités, a conduit les promoteurs de PEGASUS à construire un lieu d'action virtuellement externalisé. Cette externalisation est matérialisée, voire territorialisée, par un campus numérique qui emprunte aux membres du consortium des pratiques et des acteurs sans chercher explicitement à influer sur les pratiques sociales de chaque établissement. À l'inverse, au sein des campus numériques TEMIS et ENFI (lesquels portent sur le développement d'un portail de services), la stratégie de développement poursuivie par les équipes $a d$ hoc situe les objectifs de dissémination des pratiques innovantes à l'intérieur d'un même établissement. Les deux projets mettent en effet des outils à disposition des acteurs présents dans l'institution, puis les accompagnent. Une différence mineure peut être observée entre les deux portails : les promoteurs d'ENFI souhaitent en

18. Par « artisanat », nous sous-entendons le rapport du projet à la pratique de l'enseignement. Ce qui se traduit par la question: existe-il une séparation entre la conception et le fonctionnement-exécution? L'objectif n'est pas d'opposer des modes de production des biens et des services, mais de questionner les possibles différenciations observées et d'en comprendre les possibles complémentarités.

19. Bonami M., Garant M. (Dir.), Systèmes scolaires et pilotage de l'innovation, Bruxelles, De Boeck, 1996.

20. Le DAEU donne l'équivalent du baccalauréat français pour des adultes en reprise d'études. 
plus valoriser leur action en communiquant sur leurs pratiques innovantes. En tant que portail externe, leur objectif est une valorisation, vis-à-vis de partenaires, des pratiques innovantes initiées au sein de l'établissement. L'équipe ad hoc du campus numérique SCENARISUP vise, elle, le développement de nouvelles pratiques de création et de gestion documentaire, ce aussi bien à l'intérieur de son établissement d'appartenance qu'à l'extérieur, à savoir au niveau des 10 universités partenaires. CVS a pour objectif de développer une expertise helvétique qui pourra être exportée et qui permettra à la Suisse de participer au développement généralisé des formations e-learning. Ces terrains expriment de manière différenciée une même préoccupation, à savoir la reconfiguration des modes de production des biens et des services. $\mathrm{Si}$ leur cible, leur mode d'action sont singuliers, la finalité est commune : mettre en place des collectifs engagés dans la conception et/ou la mise en œuvre de produits et de services. Les dynamiques rationalisatrices sont fortement présentes (exemple de SCENARISUP) et se traduisent par la mise à disposition des enseignants d'outils de rationalisation de la production de produits que sont les ressources éducatives.

Pour ce qui concerne le choix de l'organisation du travail (niveau méso), les cinq campus numériques étudiés semblent positionnés selon deux options :

- la «préservation» des pratiques d'enseignement dans les établissements d'enseignement supérieur. C'est le cas dans TEMIS et ENFI. Cette préservation n'est pas synonyme d'inertie. L'échelle à laquelle s'opèrent les modifications des pratiques explique leur faible lisibilité au niveau d'une institution. À l'échelle de l'enseignant-chercheur, acteur du changement, le changement peut-être important sans pour autant qu'il se traduise par des reconfigurations en dehors de son propre espace-temps de l'action pédagogique.

- la rupture avec ces pratiques, avec l'instauration d'une division des tâches et d'une standardisation des procédures. C'est le cas au niveau de PEGASUS, avec son dispositif où les fonctions enseignantes sont éclatées et prédéfinies, et de SCENARISUP, qui tente de mettre en place, par établissement, un système commun de production et de gestion des contenus. Dans ce dernier cas, en dépit de l'objectif initial qui consistait à articuler démarche industrielle (quantité importante de supports de cours traités) et démarche « artisanale» (qualité de la relation avec l'enseignant, amélioration pédagogique «durable», formation des enseignants, recherche d'outils orientés auteurs), il ressort aujourd'hui, semble-t-il, que le projet est principalement lié à l'industrialisation de la création de documents pédagogiques (standardisation et massification des documents, objectif d'optimisation et de rendement). À une autre échelle, l'un des objectifs du CVS est le développement d'une culture e-learning au-delà d'une approche traditionnelle qui présuppose que la transposition du matériel pédagogique est facile et s'effectue en relation avec une cellule technique d'appui à la médiatisation.

Notre troisième niveau d'analyse concerne le rapport que la stratégie du campus numérique vise à entretenir avec l'enseignant-chercheur (niveau micro). PEGASUS, dès la conception de la configuration de son dispositif pédagogique et des services aux apprenants, est parvenu à sa forme quasi définitive. Les changements mineurs 
intervenus pour améliorer le fonctionnement du dispositif (tels que le changement de plate-forme, ou le « reparamètrage » du tutorat) ne sont pas issus des enseignants, mais de l'équipe du projet, soucieuse de respecter la viabilité économique du dispositif. Dans cette situation, les enseignants sont confrontés à un dilemme : adhérer à la logique du dispositif ou abandonner.

Pour les campus numériques TEMIS et ENFI, la stratégie visant l'implication des acteurs choisie par l'équipe ad hoc du projet repose sur la participation collaborative des enseignants. Les portails sont construits avec eux et pour eux. Leur avis compte et ils sont aidés par l'équipe ad hoc dans leur travail quotidien d'utilisation des ressources mises à leurs disposition (TEMIS) ou d'alimentation des sites pédagogique (ENFI). Au-delà de ce qui pourrait paraître comme une volonté de mutualisation, la dynamique qui anime ces projets relève davantage de la volonté de la construction d'un territoire commun d'action. En cela, la mutualisation est une modalité et non une fin en soi.

Dans le cas de SCENARISUP, l'acteur qui est supposé engendrer l'innovation est l'auteur de contenu, à savoir l'enseignant-chercheur. Cependant, un acteur intermédiaire intervient dans le processus de conception des contenus : la Cellule d'Ingénierie Pédagogique de l'Université, qui assiste l'auteur dans son processus de création. Ainsi, le problème qui se pose est la « dépossession » du texte fourni par l'auteur, liée à une logique de standardisation et de massification des contenus (gestion documentaire). Dans ce cadre, l'auteur (enseignant) ne dispose que d'un pouvoir consultatif sur son œuvre. Il reste tributaire de la stratégie de conception et de gestion documentaire définie par la Cellule d'Ingénierie Pédagogique de l'Université.

GIRAFE accompagne l'innovation qui est pensée depuis des centres d'expertises qui sont en appui aux projets des enseignants. L'analyse laisse apparaître une difficulté dans ces pratiques résultant du degré de reconnaissance de ces cellules par les acteurs, les institutions. Ce constat pose explicitement la question de la relation entre la légitimité de ces structures, leur positionnement et leur propre posture visà-vis des enseignements.

La description et l'analyse macro, méso et micro de ces différents campus donnent à voir semble-t-il une réalité empirique diverse. Peut-être est-il possible de distinguer, dans une première approche, deux grands types de campus :

- ceux qui cherchent à demeurer " proches » des pratiques antérieures, ou du moins - car tous semblent finalisés (serait-ce implicitement) par des objectifs de changements de pratiques - ceux qui s'attachent à s'enraciner dans les organisations et pratiques traditionnelles de l'enseignement supérieur (ENFI ; TEMIS ; Girafe) ;

- ceux qui, au contraire, reposent d'emblée sur des organisations et des pratiques hétérogènes à celles-ci, comme PEGASUS - ou SCENARISUP, proposant sans doute, au moins sur le plan des intentions, un compromis entre ces deux approches? 
Il demeure qu'après un minimum de quatre années d'existence de ces terrains, les premiers entretiens réalisés auprès des différents acteurs indiquent que, pour l'instant, l'introduction des TIC par tous ces campus numériques ne semble pas avoir encore engendré de processus d'innovation pédagogique. Tout se passe comme si, par-delà l'hétérogénéité des projets, des démarches ou des postures, s'attestait une même absence de changement de pratique pédagogique. Que la démarche soit inter- ou intra-établissement, qu'elle repose sur un fonctionnement artisanal ou bien au contraire industriel, qu'elle soit au service des enseignants ou plutôt au service du dispositif lui-même, dans tous les cas les projets semblent se heurter à ce même «verrou » que paraît constituer l'enseignant-chercheur. Les valeurs, la culture, l'identité des enseignants-chercheurs participeraient au maintien de cette stabilité, expression de pratiques routinisées dont la mise en mouvement ne pourrait résulter de la simple présence de technologie, ni même d'une cellule TIC. Une reconfiguration des espaces-temps sociaux de l'action serait une modalité de la transformation des pratiques, de l'innovation.

Comment donc rendre compte à la fois de cette hétérogénéité des terrains et de leur identité (au moins apparente) du point de vue du changement (ou de son absence) ? Il est sans doute ici possible de mobiliser un thème comme celui de l'apprentissage - en l'occurrence l'apprentissage des enseignants-chercheurs -, et de souligner que dans tous les cas évoqués, ceux-ci n'apprennent pas (au sens constructiviste du terme) : soit parce que le projet est «trop proche» de leurs pratiques (il ne les remet pas en cause), soit parce qu'il en est « trop loin» (il ne parvient pas à être en prise avec elles). Certes, mais comment penser cette pédagogie elle-même de l'enseignant-chercheur ? Comment problématiser les conditions d'un authentique apprentissage de nouvelles pratiques pédagogiques? Nous proposons ici une approche en termes de mondes de la formation; elle va nous conduire à penser l'apprentissage et le changement comme hybridation de différents mondes (ou encore comme un espace « inter-monde »).

\section{Quelles hybridations des mondes dans les campus numériques?}

Dans la lignée de Thévenot, Boltanski, Derouet, Bernard Blandin a proposé de distinguer, dans une perspective sociologique, quatre mondes sociaux de la formation. Ces quatre mondes sont distingués à partir de deux critères : la taille du public visé (grand ou petit nombre); la nature de la prestation (produit ou service).

Blandin caractérise ces 4 mondes par leur valeur principale ainsi que par les « figures idéales » du formateur et du concepteur associés. 


\begin{tabular}{|c|c|c|}
\hline & Petit nombre & Grand nombre \\
\hline Service & $\begin{array}{c}\text { Service de proximité (exemple : } \\
\text { les Ateliers de Pédagogie Personnalisée } \\
\text { - APP) }\end{array}$ & $\begin{array}{c}\text { Service global (exemple : } \\
\text { Campus virtuel) }\end{array}$ \\
\hline Produit & $\begin{array}{c}\text { Artisanat (exemple : } \\
\text { enseignement supérieur } \\
\text { traditionnel) }\end{array}$ & $\begin{array}{c}\text { Industrie (exemple : } \\
\text { Centre National d'Enseignement } \\
\text { à Distance - CNED) }\end{array}$ \\
\hline
\end{tabular}

Tableau 1. Les quatre mondes de la formation (d'après Blandin, 2002)

\begin{tabular}{|c|c|c|c|}
\hline Monde & Valeur principale & Figure du formateur & $\begin{array}{c}\text { Figure } \\
\text { du concepteur }\end{array}$ \\
\hline Artisanat & $\begin{array}{c}\text { Pédagogie (centrée } \\
\text { sur les savoirs) }\end{array}$ & $\begin{array}{c}\text { Formateur- } \\
\text { enseignant }\end{array}$ & $\begin{array}{c}\text { Ingénieur } \\
\text { de formation }\end{array}$ \\
\hline $\begin{array}{c}\text { Service } \\
\text { de proximité }\end{array}$ & $\begin{array}{c}\text { Productivité } \\
\text { de la formation }\end{array}$ & Pas de formateur & $\begin{array}{c}\text { Didacticien, expert } \\
\text { en contenu }\end{array}$ \\
\hline Service global & Efficacité du service & Tuteur-expert & $\begin{array}{c}\text { Concepteur d'outils } \\
\text { d'accompagnement }\end{array}$ \\
\hline
\end{tabular}

Tableau 2. Les caractéristiques des quatre mondes de la formation (d'après Blandin, 2002)

Dans cette perspective, Blandin peut définir et distinguer deux types de changements susceptibles d'affecter les campus numériques et les organisations dans lesquelles ils se déploient. Un premier changement, que l'on peut considérer comme relativement mineur, se caractérise ainsi: les TIC sont introduites à l'intérieur d'un monde social, sans le transformer radicalement (au niveau de ses valeurs par exemple), même si, précise Blandin, l'introduction d'objets techniques nécessairement «transforme les pratiques et les compétences» (p. 208). L'auteur identifie un second changement, plus significatif ; il se produit quand les dispositifs mis en œuvre combinent des mondes différents. Blandin semble précisément associer les campus numériques à ce type de changement: "Cette forme d'évolution est encouragée en France par les appels à projets "Campus numériques" du ministère de l'Éducation nationale. Le dispositif produit, baptisé "présentiel amélioré", associe le monde de 1'“artisanat" et le monde du "service global". Ce couplage recèle des difficultés potentielles au-delà des phases expérimentales, qui se cristalliseront autour des différences d'appréciation des aspects économiques par les acteurs...» (p. 209). Ce « couplage » se produit-il sur nos terrains ?

Les terrains observés ne peuvent être situés uniquement dans l'un ou l'autre monde. À la lueur des premiers entretiens il semble possible de considérer que 
chacun des terrains exprime de manière privilégiée un monde à l'exception de PEGASUS qui peut-être vise à la fois un service, le tutorat, et des produits pour des grands nombres. Sur chacun des terrains s'atteste de manière privilégiée soit des changements de type 1 , soit des difficultés de changement de type 2 , expression de difficulté d'hybridation.

Enracinés dans les pratiques de l'enseignement supérieur, TEMIS et ENFI semblent relever du monde de l'artisanat et d'un changement de type 1 (mineur). Comme nous l'avons suggéré, SCENARISUP relève, lui, d'une combinaison, au moins potentielle, entre l'industrie - portée par l'outil Scenari et la démarche associée - et l'artisanat des universités visées par le projet. Les centres de ressources associées au projet (et au cœur des difficultés apparemment) ne sont-ils pas précisément chargés d'assumer, comme ils le peuvent, cette combinaison ou hybridation, signe d'un (potentiel) changement de type 2 ?

Ce premier cadre d'analyse exprime à la fois l'hétérogénéité de nos terrains - ils relèvent de mondes distincts - et leur (possible) identité du point de vue de l'absence de changement (du moins de changement significatif). Cette même absence semble procéder, en effet, soit de ce que le campus numérique introduit du changement (mineur) à l'intérieur d'un même monde (ENFI, TEMIS, GIRAFE ?), soit de ce qu'il relève d'un monde qui ne s'hybride pas (ou peu) avec l'artisanat caractérisant l'enseignement supérieur (PEGASUS, SCENARISUP ?). Les limites de cette proposition de catégorisation sont rapidement atteintes dès lors qu'il n'est plus possible d'inscrire un terrain dans un seul monde. La visée bi-dimensionnelle (petit nombre - grand nombre / produit - service) de cette approche ne rend pas compte de processus plus complexes qui empruntent leurs composantes à plusieurs mondes. L'atteinte des limites de cette catégorisation questionne la recherche au plan théorique aussi bien qu'opérationnel, et nous conduit au terme de cette première analyse empirique à penser l'innovation pédagogique comme l'hybridation des mondes, c'est-à-dire comme un processus et non pas comme un résultat catégorisable dans une telle matrice. Selon cette lecture, c'est en effet cette hybridation qui rendrait possible l'apprentissage des enseignants-chercheurs et le changement des pratiques pédagogiques.

À ce stade de notre analyse, nous rejoignons pour partie les analyses de Miladi21 qui montrent que l'incertitude introduite par les campus numérique (nouveaux partenariats, nouveaux acteurs...) conduit les acteurs - en particulier les enseignants - à développer des logiques individuelles qui débouchent sur une division du travail assez forte : 1) division horizontale entre acteurs techniques (réalisateurs multimédias présents notamment au sein des cellules TIC) et enseignants; 2) division verticale entre comité de pilotage et « exécutants » (enseignants compris). Cette analyse exprime la dimension rationalisatrice de l'industrialisation que nous retrouvons dans la dynamique de mise en œuvre des campus numériques. Cette séparation concerne deux mondes, ceux de la technique (production de ressources)

21. Miladi, S., «Les campus numérique: le paradoxe de l'innovation avec les TIC» in Distances et Savoirs, 2006. 
et ceux de la pédagogie que concrétise la situation d'apprentissage. Si les campus portent en leur sein des visées idéologisantes comme nous l'avons rappelé en introduction, ils ne semblent pas susciter en tant que tels l'innovation. Des opérations de traduction sont à l'œuvre, opérations qui suscitent plus ou moins explicitement des reconfigurations des schèmes d'actions des acteurs et questionnent leur valeur et leur culture professionnelles. Ces reconfigurations supposent la création d'intermondes, du moins c'est l'hypothèse que nous formulons et que nous proposons de discuter dans la suite de cet article. Cette séparation initiale des mondes serait-elle un préalable à la construction de ces inter-mondes? Différencier pour mieux relier ensuite pourrait être l'une des composantes de cette dynamique de changement.

\section{Passage des mondes au territoire de l'action}

Les mondes évoqués dans la précédente partie de cet article sont présentés comme une catégorisation, une clé de lecture de pratique. Ils expriment des modes organisationnels sous-tendus par des valeurs, des figures d'acteurs et des finalités explicites et orientées par une vision économique de la production des biens et des services. En cela, ils sont vecteurs de différenciation, de spécification de modes d'action. Nous les considérerons comme tels sans chercher à les opposer au plan de leurs valeurs, de leurs cultures, mais en identifiant ce qui fait lien entre ces mondes et par quels mouvements, ces mondes interagissent, s'interconnectent, se potentialisent, s'hybrident, se transforment les uns les autres, pour créer des situations jusqu'alors inédites pour les acteurs, signes de l'existence d'une innovation. Mais, comme le souligne Affergan (1997, p. 117)22, «les mondes contemporains s'usent aussitôt nés ». Notre intérêt se dirige vers l'étude d'une construction médiatrice et médiatisée que nous nommerons provisoirement un « inter-monde » et qui organise, contient ces mouvements. Nous la qualifions de médiatrice tant par les fonctions communicationnelles qu'elle est susceptible d'assurer entre les acteurs, et par son statut de «médium », de «milieu », exprimant ainsi quelque chose qui n'est pas encore advenu, et quelque chose qui n'a pas encore disparu.

L'objectif de cette troisième partie est d'aller au-delà d'une catégorisation statique pour comprendre la dynamique de recombinaisons de ce qui fondait antérieurement les pratiques de la formation, une organisation spatio-temporelle et sociale. Nous proposons d'éclairer notre problématique par la compréhension des reconfigurations des temps, des espaces de l'action pédagogique. Pour ce faire nous convoquons la notion de territoire en sa qualité de témoin de la construction d'une identité collective, témoin "d'une appropriation à la fois économique, idéologique et politique de l'espace par des groupes qui se donnent une représentation particulière d'eux-mêmes, de leur histoire, de leur singularité ») (Di Meo, 1998, p. 38 $)^{23}$. Penser cet «inter-monde», revient, du moins c'est notre posture, à

22. Affergan F., La pluralité des mondes, vers une autre anthropologie, Paris, Albin Michel, 1997.

23. Di Meo, G. (1998) Géographie sociale et territoire, Paris, Nathan, U, 1998. 
comprendre les dynamiques de construction territoriale, en tant qu'espace physique et imaginaire. Par exemple, lorsqu'une cellule TICE (ou, selon les cas, une Cellule d'Innovation Pédagogique) d'un établissement évoque la difficulté à travailler avec des enseignants parce que cela remet en cause leur pratique, nous voyons là une discordance, une potentielle dissonance, entre les territoires de l'action tels qu'elle les imagine, et les territoires de l'action tels que les acteurs les vivent. Le monde industriel d'un côté, vecteur d'une rationalisation, et celui de l'artisanat de l'autre et au final l'absence d'un territoire imaginé, représenté. Cette absence d'imaginaire expliquerait, comme le souligne Guist-Desprairies ${ }^{24}$, la difficulté des acteurs, dans leur dimension individuelle et collective à adhérer à l'idée même de changement. L'incapacité des acteurs à se projeter dans ces nouvelles reconfigurations spatiales, temporelles et sociales constituerait un blocage à la transformation des pratiques. Lors des entretiens, l'un des acteurs de TEMIS indique que l'idée même de solliciter la Cellule d'Innovation Pédagogique ne lui est pas venue, alors que les besoins liés à l'activité pédagogique qu'il souhaitait mettre en place pouvaient être gérés par l'objet technique en relation avec les acteurs de la cellule. Le terrain SCENARISUP rencontre cette même difficulté d'expression d'une tension entre la production industrielle de contenu et le changement organisationnel induit par cette nouvelle organisation de la production documentaire.

Après un rappel des notions relatives au territoire, nous verrons comment les espaces-temps, les collectifs et les objets participent de cette reconfiguration territoriale, co-émergence et co-construction de pratiques sociales.

\section{Pourquoi s'intéresser à la notion de territoire ?}

Le territoire et le dispositif de formation ont en commun dans leur définition originelle l'assignation, voire l'aliénation (des références communes sont faites à Foucault). Pensées comme entités symboliques contenantes qui fondent leur action sur la contrainte, elles ne peuvent être considérées aujourd'hui comme un déjàlà inaliénable, imprescriptible, forme propriétaire d'organisation sociale légitimée. Ces constructions sont à la fois un produit du social organisateur de l'action, et une entité sujette à transformation par les conséquences de l'action. Elles sont un produit et un lieu de production, contraignantes et habilitantes (Giddens, 1987) ${ }^{25}$.

Les territoires symboliques dans leur acception socio-anthropologiques expriment des dynamiques sociales de mise en lien et de mise en sens, proches selon nous des recombinaisons à l'œuvre dans les processus d'innovation. Cette notion de territoire peut être fertile pour la compréhension de notre objet en cela qu'elle propose une analyse de l'action sociale et politique des sociétés (Di Meo, 1998 , p. 40 $)^{26}$. Pour cet auteur, les territoires sont le lieu de l'action et objet d'une

24. Giust-Desprairies F., L'imaginaire collectif, Paris, Erès, 2003.

25. Giddens A., La constitution de la société, PUF, 1987.

26. Di Meo G., Les nouveaux territoires du social, APSAM, 2001. 
appropriation sociale des conditions de production de l'action. C'est en leur qualité d'objet construit et de lieu de l'action que cette notion de territoire est convoquée dans cette analyse. Cette idée même de création d'un inter-monde, d'un lieu/objet de l'entre-deux, par les individus eux-mêmes via des interactions est retenue comme interprétation du possible processus d'innovation voulu, initié par l'introduction des campus numériques. En son absence, il n’y aurait pas innovation.

Les objets peuvent participer à cette mise en mouvement des pratiques par leur participation au renouvellement de potentialités actualisables par les acteurs. C'est parce qu'ils interpellent les acteurs du monde artisanal, notamment dans leur rapport à la production de ressources (SCENARISUP), qu'ils sont eux-mêmes acteurs de cette construction territoriale, en appelant une nouvelle configuration territoriale, au plus exactement un nouveau rapport à l'acte de production de ressources et à la ressource en tant que produit autonome, vis-à-vis duquel l'auteur peut se sentir dépossédé.

Cette dimension interactionniste située est centrale pour aller au-delà de l'approche planificatrice sous-tendue par le déploiement des campus numériques. Elle cherche à comprendre la dynamique d'effectuation de l'action. Beguin et $\operatorname{Clot}^{27}$ formulent explicitement notre projet d'analyse lorsqu'ils écrivent " D'autant que l'agent aura beau tout planifier, envisager les alternatives entre lesquelles choisir à chaque étape, l'accomplissement de l'action ne pourra être la simple exécution d'un plan. Il faudra s'ajuster aux circonstances, traiter les contingences, agir au bon moment en saisissant les occasions favorables ». Dans cette perspective, la planification préalable et prescriptive ne serait pas opérante. Le plan est un produit co-construit par les collectifs. Il est construit pour et dans l'action. Les premières observations montrent explicitement que les dispositifs en tant qu'objets construits ne suffisent pas à développer leurs usages. Leurs qualités techniques, ergonomiques ne peuvent être convoquées pour expliquer les non-usages. L'exemple de TEMIS nous le confirme lorsque l'un des utilisateurs reconnaît une facilité d'usage de l'outil, alors qu'un autre déclare ne pouvoir l'utiliser. Cela étaye l'idée de l'existence d'un processus d'appropriation singulier au cours duquel les acteurs transforment les potentialités en possibilités, ou ne les transforment pas.

L'analyse approfondie de cette divergence laisse apparaître d'autres facteurs, et tout particulièrement la dimension temporelle. Constat qui renforce l'analyse développée par Beguin et Rabardel, et par Jullien notamment sur les limites de la prescription des concepteurs de ces dispositifs qui ne peuvent avoir comme objectif la construction d'un produit fini, une forme qualifiée d'idéale. À ce propos Jullien invite, "au lieu de construire une forme idéale qu'on projette sur les choses, [à] s'attacher à détecter les facteurs favorables à l'œuvre dans leur configuration; au lieu donc de fixer un but à son action, se laisser porter par la propension; bref, au lieu d'imposer son plan au monde, s'appuyer sur le potentiel de la situation " (Jullien, 1996, 32) ${ }^{28}$. Ainsi, la mise en mouvement par lequel sont transformées les

27. Op. cit. p. 37

28. Jullien F., Traité de l'efficacité, Le Livre de Poche, nº 4292, 1996. 
pratiques résulterait de la capacité d'un collectif à définir un imaginaire commun qui permettrait la projection, ce «jeter en avant» symbolique imaginé, sorte d'autorisation à se mouvoir, et d'un ensemble de possibles structurant un potentiel qui serait actualisé dans un espace-temps social recombiné. Le territoire symbolique par sa dynamique et en sa qualité de construction sociale rassemble ce que nous cherchons à montrer, à savoir que la transformation des pratiques sociales comme traduction de l'appropriation par des collectifs d'un objet technique qu'est le campus numérique suppose à la fois un lieu et une dynamique, le territoire et la territorialisation. Cette notion territoriale, parce que Deleuze et Guattari nous invitent à la penser dans sa dynamique de déterritorialisation/reterritorialisation, nous permet de concevoir l'analyse de la construction des pratiques comme l'articulation entre des espacestemps sociaux éclatés dont la recombinaison conduit à des nouvelles territorialités, reconnues et légitimées par le collectif. Cette dimension sociale inscrite dans le territoire et la territorialisation est intéressante par leur fonction médiatrice, le rapport à l'altérité qu'il instaure pour leur construction (Di Meo, 2001, p.19).

\section{Le territoire symbolique : lieu de construction de « l'inter-monde», lieu de situation}

Le territoire peut être considéré comme un construit résultant de la rencontre entre les individus et leurs réseaux et les stratégies collectives, voire communautaires. Construction à laquelle participerait des « tiers instruits », des passeurs, des courtiers (cf. Moeglin). Ce que d'autres auteurs nomment «acteurs d'interface», sans doute à même de participer à des nouvelles mises en relations d'entités initialement hétérogènes.

Cette construction implique une redistribution de la cognition pour la réalisation de l'action, ce que Bertacchini et Girardot ${ }^{29}$ nomment l'intelligence territoriale. Posture qui nécessite de penser au-delà du local, de «l'espace classe», pour développer une pensée plus systémique qui dépasse les frontières de son territoire d'action routinier, dans lequel s'inscrivent les habitus. Cette redistribution des frontières de l'action requiert une aptitude toute particulière de mise en lien d'acteurs et d'action et de délégation à l'autre d'une partie du processus de production de l'acte éducatif. La considération de l'altérité devient centrale et convoque conjointement la notion de confiance. En effet, qui dit délégation, décharge sur l'autre d'une part de l'activité, traduit l'acceptation d'un lâcher-prise vis-à-vis de ces propres pratiques et la construction d'un objet commun ainsi que des modes de régulations spécifiques. Le territoire de l'action évolue en cela que la nature même de l'activité évolue,

29. Bertacchini et Girardot définissent l'intelligence territoriale comme un « un processus informationnel et anthropologique, régulier et continu, initié par des acteurs locaux qui s'approprient les ressources d'un espace en mobilisant puis en transformant l'énergie du système territorial en capacité de projet », Information, Savoirs, Décisions et Médiations 26, 2006. 
quantitativement et qualitativement. Quantitativement, car les activités antérieures ${ }^{30}$ demeurent mais sont distribuées temporellement, spatialement et socialement de manière différente et qualitativement car les critères même d'efficience d'une activité sont modifiés. Cette dynamique traduit un processus spécifique de délocalisation/ relocalisation au sens où l'unité originelle de réalisation de l'action délie ses composantes spatio-temporelles pour relocaliser dans un autre temps et/ou un autre espace les activités antérieures. Reprenons cette analyse à l'aide d'un exemple. La sortie du monde de l'artisanat impose l'acceptation d'exposer à autrui ses propres pratiques pédagogiques, que cela concerne les modalités, les objectifs, les ressources utilisées. L'autre peut dès lors poser un regard sur une pratique légitimement cachée. Entrer dans un monde industriel pose la rationalisation comme principe directeur de l'action planificatrice qui dépossède pour partie l'enseignement d'un pouvoir initial. Activité nouvelle qui de fait redéfinit le territoire symbolique de l'action sociale. Autrement dit, cela revient à construire son activité dans une relation à l'autre qui n'est pas uniquement l'apprenant, mais également d'autres acteurs du système. Le lieu même de socialisation de l'action qui est traditionnellement la salle de cours, ou de travaux dirigés, se déplace vers des lieux de discussion, négociation, construction, par exemple les cellules TICE ou d'Innovation Pédagogique.

Considérant comme point de départ l'existence de pratiques sociales routinisées qui assurent à la fois le fonctionnement des systèmes et une sécurité ontologique des acteurs qui s'engagent ainsi dans l'action (Giddens, 1987), le changement vu comme perturbation de cet état d'équilibre initial, suppose une " phase » spécifique que nous nommons « phase de déliance» (Bolle de Bal) ${ }^{31}$. C'est au cours de cette phase qu'il y a remise en cause des pratiques actuelles, antérieurement situées culturellement et sociologiquement. Phase de lâcher-prise qui permettra de laisser émerger des espaces de jeu, de recombinaison des liens pour une construction d'une nouvelle reliance, une nouvelle mise en liens, mais aussi en sens. L'analyse de ce processus considéré comme une délocalisation permet la séparation des composantes spatiales et temporelles de l'action. Délier le temps, l'espace et le social, autorise à les penser dans leur dynamique singulière de reconfiguration.

L'insécurité inhérente à cette situation de fragilisation peut engendrer des comportements dits de résistance ou au contraire d'euphorie innovatrice. Dans l'un et l'autre cas, cette phase doit être contenue pour assurer la transition des acteurs et du système. Les termes initiaux et finaux de cette phase ne peuvent être explicitement définis de manière causale, déterministe. C'est un état, une situation spécifique dont l'émergence peut-être repérée par l'observateur. L'anomie est sans doute plus aisément repérable que la mise en mouvement. Le mouvement n'est pas réduit à la seule « injection» (voire injonction) de techniques ou de volonté. C'est là où la notion de territoire peut être mobilisée pour comprendre l'intervention des

30. Nous signifions par activités antérieures: la préparation d'activités d'apprentissage (exemples des cours, des TD, etc.), la définition des modes d'évaluation, etc.

31. Bolle de Bal M., (ed.), Voyages au cour de sciences humaines. De la reliance, Paris, L'Harmattan, 1996. 
différentes échelles de la construction territoriale (du local au global et inversement, deux mouvements qui peut-être ne se rencontrent pas). Le territoire emprunterait dans ces dimensions concrètes et imaginées à plusieurs mondes.

Pour revenir aux terrains observés dans le cadre du projet ERTe, ces éléments ouvrent vers la formulation d'une nouvelle hypothèse : l'innovation pédagogique suscitée par les campus numériques requiert la construction d'une nouvelle territorialité. L'absence de changement serait consécutive à des processus d'aterritorialité, consécutive à l'anomie territoriale. La difficulté à reconfigurer des territoires tient pour partie à l'implication de partenaires appartenant à des mondes aux références multiples (J.L. Derouet et al., 1999) ${ }^{32}$. La notion « d'inter-monde » nous autorise à considérer ce construit intermédiaire qui dans une dynamique qui emprunte à la fois à la continuité des pratiques et leur transformation, construit de nouveaux territoires de l'action. Avant de devenir habilitante, cette construction requiert de la part des acteurs l'explicitation des valeurs et règles qui structurent leur action, tant à l'échelle de l'action locale que des références globales portées, évoquées par l'institution originelle, du moins par le territoire d'origine. Les observations actuellement réalisées sur les différents terrains tendent à montrer qu'en l'absence de ces constructions intermédiaires, le changement durable ne pourrait être initié. Il n'est demeure pas moins que des pratiques peuvent se développer mais en extériorité $\mathrm{du}$ quotidien des acteurs. Localisation qui ne les inscrit pas durablement dans le temps, car elles ne participent pas à une reconfiguration territoriale.

Identifier les lieux, en tant qu'espace-temps sociaux de l'explicitation est vecteur de compréhension de la dynamique à l'œuvre. Ces lieux de l'action des collectifs demandent une intention particulière pour en comprendre la co-émergence et comprendre comment le territoire se construit comme espace d'intéressement, résultante d'une relation d'intersubjectivation de l'action, nécessaire à la coordination de l'action. Ce n'est pas un simple lieu d'édification des principes, mais bien un lieu de co-construction légitimatrice de principes d'action et de régulation en cours d'action. Il est le lieu de l'élaboration de situations nouvelles, c'est-àdire des complexes "de ressources et de contraintes, qui peuvent toutes le cas échéant jouer un rôle significatif sans pour autant que ce rôle soit nécessairement réductible à un jeu de représentations mentales préalablement objectivées dans les appareils cognitifs... les actions sont toujours socialement et physiquement situées, et la situation est essentielle à l'action » Visetti (1989) ${ }^{33}$. Ce développement conduit à proposer l'hypothèse que cet «inter-monde » dans sa fonction médiatrice et médiatisée résulterait de la construction de situation nouvelle. Elle résulterait de la création d'un espace d'intéressement entre les membres d'un collectif, et en cela serait à la fois le lieu et le produit de l'expérience. Nous rejoignions les propos de Quéré sur cette dimension spécifique de la situation, lorsqu'il écrit que "l'intérêt de cette perspective est d'insister sur le caractère non prédéfini des situations, des

32. Derouet J.-L. et al., L'école dans plusieurs mondes, Bruxelles, De Boeck, 1999.

33. Visetti Y.-M., Constructivismes, émergences : une analyse sémantique et thématique, CREA-CNRS, 1989. 
actions, des objets et du cadre de travail : ceux-ci acquièrent leur définition précise dans une dynamique de codétermination orientée par l'activité en cours 》 (Quéré, 1997). Si au plan théorique, la cohérence de cette analyse concourt à comprendre la construction des pratiques, il reste à comprendre d'un point de vue pragmatique, cette dynamique, celle par laquelle les situations assurent une fonction d'intermédiation dans la construction de pratiques territorialisées. D'où la proposition de s'intéresser non pas à la recherche de cause, mais à la présence ou non d'un potentiel de situation, de ce qui permet au mouvement d'advenir.

\section{Le changement, dynamique potentialisatrice territorialisée}

Reprenons notre triangulation initiale « espace-temps », « collectifs, « objet ». La simple maîtrise d'outil ne suffit pas à construire les territoires de l'action et des pratiques sociales, en d'autres termes à produire de l'innovation. Prenons le cas de l'enseignant-chercheur qui dans sa posture de chercheur sollicite et mobilise fortement les techniques d'information et de communication, alors que les utilisations qu'il en fait dans sa pratique pédagogique demeurent plus ténues, parfois quasi-inexistantes. Les utilisations ne migrent pas d'un monde à l'autre, elles n'assurent pas en tant que tel l'interconnexion entre deux territoires, celui de la recherche d'une part, et celui de la pédagogie d'autre part. À tout le moins ce n'est pas la compétence technique, celle qui assure la maîtrise manipulatoire de l'objet technique qui peut suffire à définir une nouvelle territorialité. L'explication est sans doute à chercher du côté des situations, de leur singularité dans l'un et l'autre monde et de leur dynamique même de construction qui emprunterait des éléments à des mondes différents. L'hypothèse selon laquelle l'absence d'innovation résulterait de l'absence d'un processus de déterritorialisation/reterritorialisation conserve son actualité.

Ce processus peut être observé en identifiant ce que Giddens nomme dans sa théorie de la structuration, les arêtes spatio-temporelles des mondes sociaux. Cette approche constitue une piste d'analyse pour comprendre ce qui fait «frontière » entre ces différents mondes, et espaces-temps sociaux, ce qui définit les territoires, la régionalisation de l'action. Si ces dimensions spatio-temporelles contiennent l'action, cette dernière est « organisée » par un ensemble de règles et de ressources. L'enjeu alors n'est-il pas de comprendre comment ces deux axes s'articulent pour mettre en mouvement les acteurs et le système. Mouvement qui doit être analysé tant au niveau des sujets dans leur individualité que dans leur dimension collective. Les caractéristiques spatiales, temporelles et sociales qui structurent les différentes activités des acteurs sont différentes et liées à la nature même de la tâche. Sans vouloir caricaturer l'opposition entre les tâches d'enseignement et celles de recherche, les personnes interviewées sur différents terrains (TEMIS, PEGASUS), rappellent cette disjonction fonctionnelle entre le monde pédagogique et celui de la recherche. Ils expriment d'ailleurs explicitement le différentiel rythmique de la gestion temporelle dans ces deux tâches : un temps réglé, récurrent, géré par un tiers dans le cas de l'enseignement, un temps libre, construit, géré par soi-même 
dans le cas de la recherche. Il en est de même pour la spatialisation de l'activité qui se déroule dans des lieux et des conditions d'usage des lieux différents, lesquels répondent à des critères d'usage semblables au temps : lieu prescrit, lieu choisi. Cette différenciation, bien qu'établie à grands traits, illustre la présence de ces arêtes spatio-temporelles. Dans leur propre contexte, les acteurs organisent leur action, et structurent des situations efficientes qui, pour chacune des tâches « obéissent » à des règles spécifiques.

La première partie suggère l'absence d'innovation pédagogique après quatre années de mise en place des campus numériques. L'une des explications réside sans doute dans la visée déterministe de l'introduction de ces techniques. Une autre explication peut être donnée en rappelant le statut même de l'expérimentation qui ne traite pas explicitement de la transformation du structurel, entendu ici les règles et ressources habituelles de fonctionnement. Par exemple, nous observons les difficultés à poursuivre l'action engagée par une équipe au sein d'une université dans le cadre d'un projet euroméditerranéen Interreg IIIB (SCENARISUP). Ce serait l'absence d'une visée explicite de construction de nouvelles routines articulées avec l'existant qui inhiberait la pérennisation de l'action en dehors du contexte expérimental. Le contexte changerait sans modifier au fond la situation, sans doute en raison de l'absence d'un véritable processus à la fois projectif et réflexif organisateur des recombinaisons transformatrices des pratiques sociales.

Le changement, parce qu'il remet en cause cet état d'équilibre, suppose, du moins c'est notre hypothèse, l'émergence d'un désir de changer, catalyseur du mouvement.

\section{Conscience et imaginaire : vecteurs de la territorialisation}

Mais existe-t-il, chez les acteurs, un niveau de conscientisation de la nécessité d'aller au-delà de cette fonction symbolique, nécessité d'objectivation territoriale pour initier, mettre en mouvement le système, et d'accepter la remise en cause d'un sentiment de sécurité en quelque sorte routinisé ? Comment naît cette prise de conscience et cette acceptation de la mise en mouvement ? Sans doute convient-il de chercher dans les observations les indices de cette objectivation qui permettraient de comprendre comment se déroule ce processus de déconstruction/reconstruction territoriale.

L'hypothèse sous-jacente à ce propos est la nécessité de situer cette reconfiguration à la fois dans un espace-temps social "protégé » et dans une zone "proximale » et «proxémique ». Les adjectifs protégé, proximale et proxémique semblent être les trois piliers structurels qui conditionnent une dynamique de changement. L'espace protégé (Bourgeois, 1996) ${ }^{34}$ renvoie à l'édification d'un espace-temps social qui légitime la réflexion sur l'objet qu'est l'innovation, maintenant de fait une forme de sécurité nécessaire à la prise de risque consécutive à la sortie de trajectoire

34. Bourgeois E., « Identité et Apprentissage », Éducation Permanente 128, pp. 27-35, 1996. 
routinière (sortie de route à moindre risque). La dimension proximale est retenue pour introduire une notion séquentielle, du moins itérative dans le changement. Le changement ne pouvant être réalisé que par étapes correspondantes au «pas de changement » que les individus et les collectifs sont à même de réaliser. Enfin, la dimension proxémique semble convoquée dans cette dynamique pour ce qu'elle assure comme étayage auprès des sujets et des collectifs. Elle peut être reliée avec les concepts de lieu et de disponibilité de présence proposés par Giddens.

À ce stade de nos investigations, nous ne voyons pas émerger de dynamique de cristallisation par laquelle l'innovation pourrait advenir. Cette analogie faite au processus de cristallisation exprime ce processus par lequel, à partir d'éléments initiaux, se transforment les pratiques sociales. Cette absence de construction originelle est à analyser. De nombreux implicites préalables et souvent extérieurs fondent les projets de campus numérique (mieux faire pédagogique, marché concurrentiel des formations). Implicites fréquemment aliénants (car allants-de-soi) pour les acteurs et collectifs à qui sont proposés ces dispositifs.

\section{« L'inter-monde », un lieu entre intériorité et extériorité}

La fonction médiatrice de cet inter-monde, articule à la fois l'intériorité et l'extériorité des lieux physiques, symboliques et identitaires de réalisation des pratiques habituelles. En cela, le lieu même de l'innovation technique est intéressant à identifier. Dans les terrains observés nous voyons comment ces dispositifs sont initialement en extériorité avec le système initial pour lequel ils sont mis en place (le cas de PEGASUS illustre une extériorité quasi-totale). Le degré de leur extériorité est variable, et rarement situé en semi-extériorité, du moins à bonne distance des pratiques en cours. Nous ne pouvons d'ailleurs pas conclure qu'ils potentialisent une situation. Ils constituent un environnement, un élément de contexte pour les acteurs, sans être des composantes de la situation. Il ne semble pas y avoir de mouvement qui articule aux différentes échelles de l'organisation ce qui est, et ce qui est en capacité d'advenir. Un mouvement réciproque qui fait circuler de l'intérieur (au sens des pratiques incorporées) vers l'extérieur qui contient un ensemble de possibles les « composantes » du changement (valeur, désir...). Si l'objectif est l'internalisation par les collectifs d'éléments initialement externes, pour en faire leurs propres règles d'action, il ne semble pas atteint.

Cette question de territorialisation de l'action, pensée dans un rapport frontalier du dedans et du dehors de l'institution originelle peut être illustrée par des premiers éléments d'observation des terrains. Lorsque les acteurs du terrain ENFI orientent leur projet vers un portail, nous voyons comment ce positionnement extérieur organise l'avenir du projet. L'hypothèse implicite des acteurs est-elle celle du levier? Autrement dit, cherchent-ils par une communication de l'offre de formation, un positionnement vers l'externe, à interpeller l'interne quant à la validité de son offre de formation, s'exposant à la lecture concurrentielle de l'offre ? Dans ce cas, l'absence d'un collectif préalablement constitué participerait de la 
recherche de modes opérationnels pour constituer ce collectif, sollicitant un registre de crainte, d'instabilité potentielle. Il y a tentative de construction d'une nouvelle régionalisation, articulant des lieux différents. Cette tentative est potentiellement limitée par les sentiments d'insécurité que peuvent éprouver les acteurs. La carence de ce que Giddens nomme la sécurité ontologique participe sans doute de la difficulté des acteurs à s'engager dans ces dynamiques. Cette analyse corrobore l'implication de la dimension socio-affective dans les pratiques de changement (cf. dispositif transitionnel) $)^{35}$. Laquelle convoque le besoin de protection exprimé dans la notion « d'espace protégé ».

\section{Pour conclure}

C'est bien parce qu'il est impossible, du moins c'est notre choix théorique, de délier sujet, dans sa dimension individuelle et collective, et situation, qu'il nous faut penser le changement comme des mises en mouvements exogènes et endogènes. Exogènes en cela qu'ils expriment l'évolution des relations collectifs/situations et endogènes pour ce qui relève de l'évolution du sujet. Ainsi dans cette perspective, situations et agents de la situation interagissent pour la réalisation de l'action, interaction fondatrice de pratiques sociales reconfigurées. L'approche par le lien entre ces composantes ouvre sans doute vers d'autres analyses du changement. Les produits de ces interactions structurantes du changement ne sauraient être prédéterminés et ce pour plusieurs raisons. D'une part parce que l'interaction produit des changements endogènes et exogènes qui font que ce qui était projeté peut perdre son sens car la vision du monde évolue. D'autre part, parce que l'interaction produit des conséquences non-intentionnelles, non attendues qui conduisent à revoir les attendus. L'apparition des blogs d'étudiants dans un dispositif de formation, produit de l'interaction entre un collectif, celui des apprenants, peut avoir des conséquences non-intentionnelles du côté du collectif de tuteurs, de formateurs.

Penser l'innovation pédagogique comme processus de déterritorialisation/reterritorialisation revient en partie à accepter l'idée que l'innovation conduit à revoir le structurel, autrement dit les processus d'allocation des ressources et de définition des règles de fonctionnement. L'un ne peut aller sans l'autre. La mise en mouvement serait alors rendue possible par l'existence d'espace-temps sociaux et d'objets qui sont les lieux d'évolution structurelle, redéfinissant à la fois les contraintes structurelles et les pouvoirs des acteurs. L'objet devient davantage sujet de réflexivité, qu'outil à manipuler, assurant une fonction d'intermédiation entre collectifs et situation.

35. Paquelin D., Choplin H., « Nouveaux médias et innovation pédagogique : l'hypothèse d'un dispositif transitionnel », Éducational Media International, vol. 38, $n^{\circ}$ 2/3, june/september, pp. 165-174, 2001. 


\section{Bibliographie}

Affergan F., La pluralité des mondes, vers une autre anthropologie, Paris, Albin Michel, 1997.

Alter N., L'innovation ordinaire, Paris, PUF, 2000.

Beguin P., Clot Y., «L'action située dans le développement de l'activité », Activités, vol. 1 (2), 27-49, http://www.activites.org/vln2/beguin.fr.pdf, 2004.

Bissey C., Moreau J.-L., TIC et NET, Nouvelles voies pour la formation, Paris, PUF, 2003.

Blandin B., "Les mondes sociaux de la formation», in Les TIC au service des nouveaux dispositifs de formation, Éducation Permanente n 152, p. 199-211, 2002.

Bolle de Bal M., (Ed.), Voyages au cœur de sciences humaines. De la reliance, Paris, L'Harmattan, 1996.

Bonami M., Garant M. (Dir.), Systèmes scolaires et pilotage de l'innovation, Bruxelles, De Boeck, 1996.

Bourgeois E., «Identité et Apprentissage », Éducation Permanente 128, pp. 27-35, 1996.

Cros F. (dir.), Dynamiques du changement en éducation et en formation, Versailles, INRP/ IUFM, 1998.

Derouet J.-L. et al., L'école dans plusieurs mondes, Paris-Bruxelles De Boeck, 1999.

Di Meo G., Les nouveaux territoires du social, 2001, APSAM, 2001.

Geertz C., Ici et là-bas, Paris, Métailié, 1996.

Giddens A., La constitution de la société, PUF, 1987.

Giust-Desprairies F., L'imaginaire collectif, Paris, Erès, 2003.

Glikman V., «Formation à distance», in Champy P. \& Etévé C. (Dirs.), Dictionnaire encyclopédique de l'éducation et de la formation, Paris, Nathan, pp. 441-446, 1994.

Honoré B., Soigner, Paris, Seli Arslan, 2001.

Jacquinot-Delaunay G., Monnoyer L. (Dirs.), Le dispositif entre usage et concept, CNRS Hermes 25, 1999.

Jacquinot-Delaunay G., Choplin, H., « La démarche dispositive aux risques de l'innovation », Éducation permanente 152, 2002.

Jullien F., Traité de l'efficacité, Paris, Le Livre de Poche, 1996.

Kaye A., «L'enseignement à distance : un état de la question ». Perspectives, vol. XVIII, $\mathrm{n}^{\circ} 1$, pp. $41-54,1988$

Paquelin D. et al., Analyse systémique des usages des TIC pour l'apprentissage, Rapport final, CNCRE INRP, 2000

Paquelin D., Choplin H., « Nouveaux médias et innovation pédagogique: l'hypothèse d'un dispositif transitionnel » Educational Media International, vol. 38 , n 2/3, june/ september, pp. 165-174, 2001 
Peraya D., « Médiation et médiatisation : les campus virtuels » in Jacquinot-Delaunay G. et Monnoyer L. (Dirs.) Le dispositif entre usage et concept, CNRS Hermes 25, 1999.

Proulx S., "Trajectoires d'usages des technologies de communication: les formes d'appropriation d'une culture numérique comme enjeu d'une "société du savoir » in De La Vega J. et Licoppe C., Usages émergents des TI, Annales des télécommunications, 57.

Quéré L., « La situation toujours négligée », CNET Réseaux 85, 1997.

Visetti Y.-M., Constructivismes, émergences : une analyse sémantique et thématique, CREACNRS, 1989. 
